\title{
A class of invisible inhomogeneous media and the control of electromagnetic waves
}

\author{
B. Vial, ${ }^{1, *}$ Y. Liu (刘泱杰), ${ }^{1}$ S. A. R. Horsley, ${ }^{2}$ T. G. Philbin, ${ }^{2}$ and Y. Hao ${ }^{1}$ \\ ${ }^{1}$ School of Electronic Engineering and Computer Science, Queen Mary University of London, London E1 4NS, United Kingdom \\ ${ }^{2}$ Department of Physics and Astronomy, University of Exeter, Stocker Road, Exeter EX4 4QL, United Kingdom
}

(Received 19 August 2016; revised manuscript received 15 November 2016; published 13 December 2016)

\begin{abstract}
We propose a general method to arbitrarily manipulate an electromagnetic wave propagating in a twodimensional medium, without introducing any scattering. This leads to a whole class of isotropic spatially varying permittivity and permeability profiles that are invisible while shaping the field magnitude and/or phase. In addition, we propose a metamaterial structure working in the infrared that demonstrates deep subwavelength control of the electric field amplitude and strong reduction of the scattering. This work offers an alternative strategy to achieve invisibility with isotropic materials and paves the way for tailoring the propagation of light at the nanoscale.
\end{abstract}

DOI: 10.1103/PhysRevB.94.245119

\section{INTRODUCTION}

In recent years, the introduction of transformation optics has shed new light on the propagation of electromagnetic waves in complex media and has proven to be an intuitive yet powerful tool for engineering the flow of light at the subwavelength scale [1-3]. The theory is based on the invariance of Maxwell's equations under a change of coordinates, resulting in equivalent permittivity and permeability profiles that are generally anisotropic, spatially varying, and sometimes singular. Perhaps the most popular application has been an invisibility cloak, which has been realized experimentally in various frequency regimes for two-dimensional and three-dimensional setups [4-6] thanks to the development of metamaterials and advanced manufacturing techniques [7]. However, the complexity of the required material properties makes practical realization a hard task, while the use of resonant meta-atoms to reach extreme parameters results usually in a narrow frequency band of operation $[8,9]$. There is thus a critical need for other approaches to achieve invisibility at least to reduce diffraction significantly such as mantle cloaking [10], optimized dielectric covers [11,12], or by introducing gain [13,14]. Quite paradoxically, although it is a very common phenomenon in wave physics, relatively little is known regarding what does or does not cause scattering when the material properties are allowed to vary rapidly in space [15-18]. Finally, there is an ever increasing demand for controlling optical fields at the nanoscale for applications ranging from medical diagnostics and sensing to optical devices and optoelectronic circuitry [19-22]. In particular, local field enhancement is of paramount importance in phenomena such as surface enhanced Raman scattering [23,24], improved nonlinear effects [25-27], optical antennae and the control of the local density of states [28,29].

In this paper we present a general purpose method to control the amplitude and/or phase of a wave propagating in a two-dimensional inhomogeneous isotropic medium. Although we focus our attention on media that does not scatter an incident plane wave while producing a specified amplitude and/or phase, the technique might be extended to arbitrary incident fields as well as to control the scattering pattern. In

*b.vial@qmul.ac.uk addition, the method is not based on the geometrical optics approximation and is valid at every frequency.

\section{GOVERNING EQUATIONS}

We consider here linear, isotropic, lossless, and possibly dispersive materials characterized by their $z$-invariant relative permittivity $\varepsilon(\boldsymbol{r})$ and relative permeability $\mu(\boldsymbol{r})$, where $\boldsymbol{r}=$ $(x, y)^{\mathrm{T}}$ is the position vector. This medium is illuminated by a monochromatic electromagnetic wave of pulsation $\omega=k_{0} / c$, amplitude $A_{0}\left(\boldsymbol{r}, k_{0}\right)$, and phase $\phi_{0}\left(\boldsymbol{r}, k_{0}\right)$ whose electric field is linearly polarized along the $z$ axis, which is the so-called TE polarization, so that $\boldsymbol{E}=E_{z} z$. Under these conditions, Maxwell's equations can be recast as the scalar wave equation:

$$
\nabla \cdot\left(\frac{1}{\mu} \nabla E_{z}\right)+k_{0}^{2} \varepsilon E_{z}=0 .
$$

By writing the total electric field in polar form as $E_{z}=A e^{i \phi}$ ( $A$ and $\phi$ real), Eq. (1) is separated into the following two equations:

$$
\begin{gathered}
\nabla \cdot\left(\frac{A^{2}}{\mu} \nabla \phi\right)=0 \\
(\nabla \phi)^{2}-k_{0}^{2} \varepsilon \mu-\frac{\nabla^{2} A}{A}+\frac{\nabla \mu}{\mu} \cdot \frac{\nabla A}{A}=0 .
\end{gathered}
$$

The physical meaning of these two equations is well known: the first is the continuity equation for the Poynting vector, while the second is the exact eikonal equation governing the motion of the rays [30,31]. They are usually solved through setting $\varepsilon$ and $\mu$ as known quantities and then solving for $E_{z}$, i.e., $A$ and $\phi$. However, the methodology presented here allows us to fix arbitrarily two parameters and then compute the two others using Eqs. (2) and (3).

From now on we consider an incident homogeneous plane wave with constant amplitude $A_{0}$ and phase $\phi_{0}\left(\boldsymbol{r}, k_{0}\right)=$ $k_{0} \boldsymbol{n} \cdot \boldsymbol{r}$, with $\boldsymbol{n}=\left(\cos \theta_{0}, \sin \theta_{0}\right)^{\mathrm{T}}$ the unit vector defining the incidence direction. The gradient of the phase can then be written as

$$
\nabla \phi=\boldsymbol{n} k_{0}+\nabla \psi
$$


where $\psi$ is an additional phase term. If $\nabla \psi \rightarrow 0$ and $A \rightarrow A_{0}$ as $r=\sqrt{x^{2}+y^{2}} \rightarrow+\infty$, the incident wave remains plane and the material will be invisible.

\section{CONTROLLING AMPLITUDE AND PERMEABILITY}

In this section we suppose that we fix $A$ and $\mu$. Substituting $\nabla \phi$ into Eq. (2), we obtain the following Poisson equation for $\psi$ :

$$
\nabla \cdot\left(\frac{A^{2}}{\mu} \nabla \psi\right)=-k_{0} \boldsymbol{n} \cdot \nabla\left(\frac{A^{2}}{\mu}\right),
$$

which can be solved to give

$$
\nabla \psi(\boldsymbol{r})=-\frac{\mu(\boldsymbol{r}) k_{0}}{2 \pi A^{2}(\boldsymbol{r})} \int d^{2} \boldsymbol{r}^{\prime} \frac{\boldsymbol{r}-\boldsymbol{r}^{\prime}}{\left|\boldsymbol{r}-\boldsymbol{r}^{\prime}\right|^{2}} \boldsymbol{n} \cdot \nabla^{\prime}\left(\frac{A^{2}\left(\boldsymbol{r}^{\prime}\right)}{\mu\left(\boldsymbol{r}^{\prime}\right)}\right) .
$$

This shows that if we specify the quantity $\zeta=A^{2} / \mu$ over space, then the gradient of the phase changes in response to the change in $\zeta$ in the same way the electric field responds to a charge density. Substituting the above equation into (3) then determines a relationship between $\varepsilon$ and $\mu$.

In the following we further assume that $A$ and $\mu$ are dispersionless and introduce the frequency independent quantities $\alpha=\phi / k_{0}$ and $\beta=\psi / k_{0}$. Locally, the permittivity dispersion takes the form of a lossless Drude model

$$
\varepsilon(\omega)=\varepsilon_{\infty}-\omega_{\mathrm{p}}^{2} / \omega^{2},
$$

with the permittivity at infinite frequency $\varepsilon_{\infty}$ and the plasma frequency $\omega_{\mathrm{p}}$ defined as

$$
\begin{gathered}
\varepsilon_{\infty}=\frac{(\nabla \alpha)^{2}}{\mu}=\frac{1}{\mu}\left[1+(\nabla \beta)^{2}+2 \boldsymbol{n} \cdot \nabla \beta\right], \\
\omega_{\mathrm{p}}^{2}=\frac{c^{2}}{\mu}\left(\frac{\nabla^{2} A}{A}-\frac{\nabla \mu}{\mu} \cdot \frac{\nabla A}{A}\right) .
\end{gathered}
$$

The obtained permittivity is linear, spatially varying, with a $1 / \omega^{2}$ dispersion and nonlocal since $\varepsilon_{\infty}$ depends on the incidence direction $\boldsymbol{n}$. On the basis of time reversal, a plane wave coming from the opposite direction gives a total field with the same amplitude but an opposite phase as $\phi(-\boldsymbol{n})=-\phi(\boldsymbol{n})$, while invisibility is maintained for the same permittivity since $\varepsilon(-\boldsymbol{n})=\varepsilon(\boldsymbol{n})$, even if generally the amplitude and material profiles do not possess any particular symmetry.

\section{A. A special case}

There is a particular situation for which we can get rid of the nonlocality, and this happens when $\nabla \beta=\mathbf{0}$, i.e., when $\mu$ is proportional to $A^{2}$. In this case and in the ray optics approximation we retrieve a medium with unit index of refraction because $\varepsilon \rightarrow 1 / \mu$ as $\omega \rightarrow+\infty$, which is an inhomogeneous medium where all the waves travel in straight lines and without reflection. Essentially, our approach can be understood by considering this limiting case $\varepsilon=1 / \mu$ and extending it to work for all frequencies and all incidences by adding dispersive and nonlocal terms into $\varepsilon$. On the other side of the spectrum, the medium becomes singular in the quasistatic limit since $|\varepsilon| \rightarrow+\infty$ as $\omega \rightarrow 0$. This behavior is due to the fact that any permeability inhomogeneity will
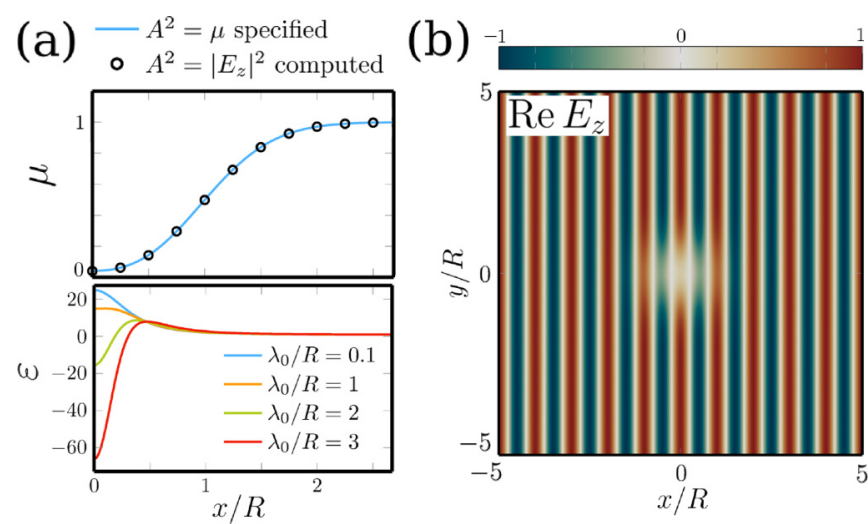

FIG. 1. Invisible material in the case $\mu=A^{2}$ with $80 \%$ damping of the field in the center. (a) Permeability (top) and permittivity (bottom) profiles along the radial direction. (b) Real part of the electric field $E_{z}$ for $\lambda_{0} / R=1$.

cause large scattering at low frequencies, and one needs large changes in the permittivity to counteract this.

Without loss of generality, we now consider the case where $\mu=A^{2}$ : this implies that the phase is exactly given by $\nabla \phi=\boldsymbol{n} k_{0}$ everywhere, i.e., the field is a plane wave with a nonuniform amplitude, and the Drude parameters simplify as

$$
\varepsilon_{\infty}=\frac{1}{\mu} \quad \text { and } \quad \omega_{\mathrm{p}}^{2}=\frac{c^{2}}{\mu}\left(\frac{\nabla^{2} \sqrt{\mu}}{\sqrt{\mu}}-\frac{\nabla \mu}{\mu} \cdot \frac{\nabla \sqrt{\mu}}{\sqrt{\mu}}\right) .
$$

We note that in this case, $\varepsilon$ is frequency dispersive but does not depend on the incidence angle, similarly to the Pöschl-Teller profile (which is reflectionless for all angles and depends on $\omega$; see, e.g., [32]) as the permittivity is analogous to the quantum potential for the Schrödinger equation.

As an example, suppose we want to obtain a field with a prescribed Gaussian amplitude $A=1-f \exp \left(-r^{2} / R^{2}\right)$, and that $\mu=A^{2}$ [see blue line on the top panel of Fig. 1(a)], with $R=700 \mathrm{~nm}$ and $f=0.8$. Note that this results in a permeability profile with values below unity, which seems to contradict our assumption of neglecting frequency dispersion for $\mu$. In practice, indeed we would likely only be able to realize the $\mu$ profile containing regions of $\mu<1$ for one single frequency. The calculated permittivity profile is shown for several wavelengths in Fig. 1(a) (bottom panel). As discussed previously, the required $\varepsilon$ is roughly equal to $1 / \mu$ for $\lambda_{0} / R=0.1$, while one needs more extreme permittivity values at longer wavelengths. We solved the wave equation (1) using a finite element method for $\lambda_{0} / R=1$, with a plane wave of unit amplitude incident from the negative $x$ axis and perfectly matched layers to truncate the domain. The real part of the electric field $E_{z}$ is plotted in Fig. 1(b) and reveals a clear damping of the field as well as no scattering and a planar wavefront everywhere. The computed square norm of the field matches the required one perfectly [see black circles in the top panel of Fig. 1(a)].

Note that the TM polarization case can be treated similarly by replacing $E_{z}$ by $H_{z}$ and swapping $\varepsilon$ and $\mu$. 

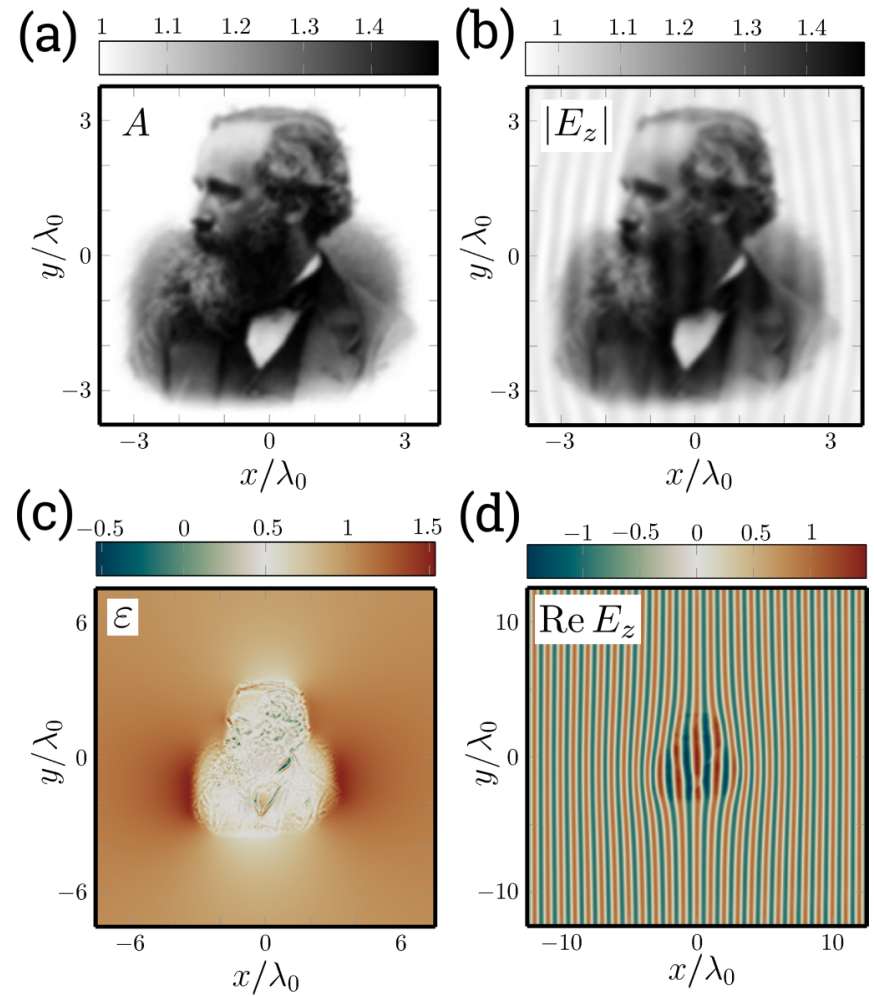

(d)

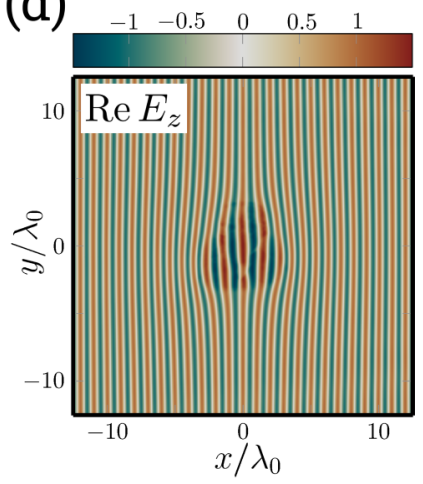

FIG. 2. Invisible material profile in the nonmagnetic case $(\mu=1)$ with arbitrary control of the amplitude. (a) Specified amplitude (from a picture of James Clerk Maxwell). (b) Computed amplitude. (c) Permittivity profile. (d) Real part of the electric field, showing the invisibility effect.

\section{B. The nonmagnetic case}

For practical reasons, we investigate the possibility of having nonmagnetic invisible profiles $(\mu=1)$. We solve Eq. (2) to obtain the phase, and the parameters for the permittivity reduce to

$$
\varepsilon_{\infty}=1+(\nabla \beta)^{2}+2 \boldsymbol{n} \cdot \nabla \beta \quad \text { and } \quad \omega_{\mathrm{p}}^{2}=c^{2} \frac{\nabla^{2} A}{A} .
$$

To illustrate the arbitrariness of the choice of the amplitude, we used a profile extracted from a grayscale image of James Clerk Maxwell depicted in Fig. 2(a), where dark values correspond to a $50 \%$ enhancement of the field, with a lateral "size" of approximately $D=6 \lambda_{0}$. The permittivity profile is displayed in Fig. 2(c), and presents small features and rapidly varying values between -0.5 and 1.5 . The real part of $E_{z}$ is displayed in Fig. 2(d), and proves clearly that the field is not a plane wave, with a retarded phase on the left and an advanced phase on the right of the inhomogeneity, but that this profile does not induce any scattering. The required field enhancement is respected as can be seen in Fig. 2(b) with no more than $5 \%$ relative error, albeit some small reflections due to numerical inaccuracies. This proves the ability of the method to devise invisible nonmagnetic media capable of shaping intricate magnitude patterns. We then investigate the angular response of this permittivity profile in terms of invisibility and amplitude control. To quantify this, we computed the scattering cross section $\sigma_{\mathrm{s}}$ normalized to the profile size $D$, along with

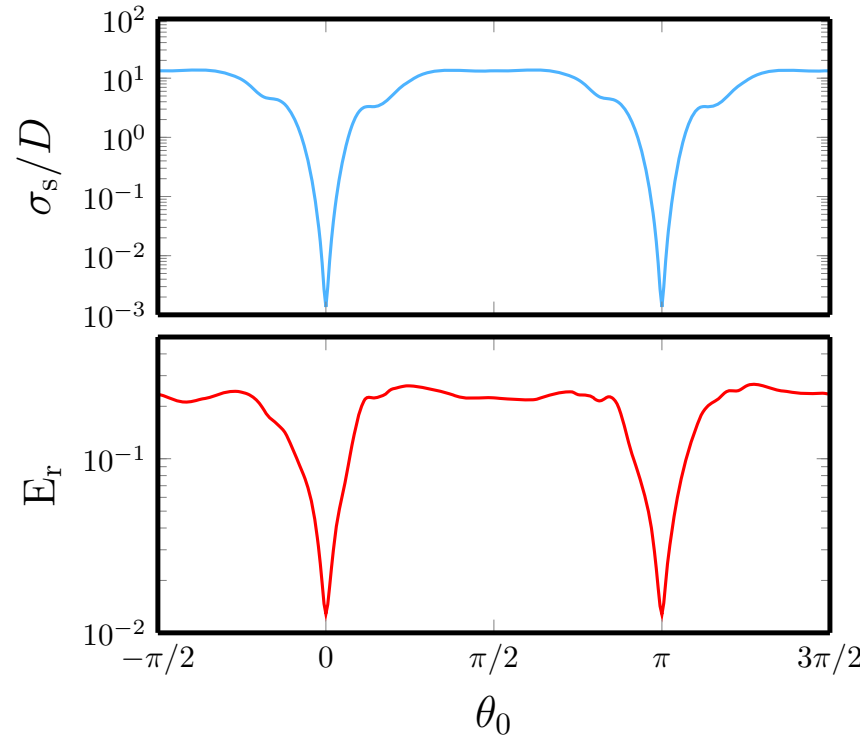

FIG. 3. Angular response of the permittivity profile of Fig. 2(c). Top: scattering cross section $\sigma_{\mathrm{s}}$ normalized to the profile size $D$. Bottom: average error on the amplitude $E_{\mathrm{r}}$ defined by Eq. (10).

the average error on the amplitude $E_{\mathrm{r}}$ defined as

$$
E_{\mathrm{r}}\left(\theta_{0}\right)=\frac{1}{S_{\Omega}} \int_{\Omega} d \boldsymbol{r}\left\|1-\frac{\left|E_{z}\left(\theta_{0}\right)\right|}{A}\right\|,
$$

where $\Omega=\left[24 \lambda_{0} \times 24 \lambda_{0}\right]$ is the computational window used [cf. Fig. 2(d)] with surface $S_{\Omega}=\left(24 \lambda_{0}\right)^{2}$. The results are plotted as a function of the incident angle $\theta_{0}$ in Fig. 3 , and clearly indicate a strong reduction of the scattering and an accurate reconstruction of the field magnitude for the reference configuration $\left(\theta_{0}=\pi\right)$ as well as for the antiparallel direction of incidence $\left(\theta_{0}=0\right)$, as discussed before. As expected, both effects are fairly narrow band due to the nonlocality of the permittivity.

\section{Metamaterial implementation}

As for a possible experimental verification of our method, we propose a metamaterial structure that approximates the permittivity profile given by Eq. (9) at $\lambda_{0}=10.32 \mu \mathrm{m}$ with $A=1-f \exp \left(-r^{2} / R^{2}\right), f=-0.9$, and $R=\lambda_{0} / 6.5=$ $1587 \mathrm{~nm}$. The resulting continuous permittivity profile is given in Fig. 4(a) and it varies between 0.044 and 2.239. To be able to reach values of permittivity smaller than unity, we use silicon carbide $(\mathrm{SiC})$, a polaritonic material that has a strong dispersion in the thermal infrared range given by the DrudeLorentz model [33] $\varepsilon_{\mathrm{SiC}}(\omega)=\varepsilon_{\infty}\left[1+\left(\omega_{L}^{2}-\omega_{T}^{2}\right) /\left(\omega_{T}^{2}-\right.\right.$ $\left.\left.\omega^{2}+i \Gamma \omega\right)\right]$, with $\varepsilon_{\infty}=6.7, \omega_{\mathrm{L}}=1.82 \times 10^{14} \mathrm{rad} \mathrm{s}^{-1}, \omega_{\mathrm{T}}=$ $1.49 \times 10^{14} \mathrm{rad} \mathrm{s}^{-1}$, and $\Gamma=8.96 \times 10^{11} \mathrm{rad} \mathrm{s}^{-1}$ [see solid and dashed cyan lines in Fig. 4(d)]. This material exhibits a dielectric to metallic transition around $\lambda_{0}=\lambda_{\mathrm{L}}=10.32 \mu \mathrm{m}$ so that $\varepsilon_{\mathrm{i}}\left(\lambda_{0}\right)=0.0009-0.0815 i$. For values greater than unity, we use potassium bromide $(\mathrm{KBr})$ with permittivity $\varepsilon_{\mathrm{KBr}}\left(\lambda_{0}\right)=2.3280$ [34]. The hybrid metamaterial structure is a $51 \times 51$ array of square unit cells of period $d=\lambda_{0} / 27=$ $377 \mathrm{~nm}$. The continuous map of Fig. 4(a) is discretized at the center $\left(x_{i}, y_{j}\right)$ of those unit cells resulting in a discrete set of 

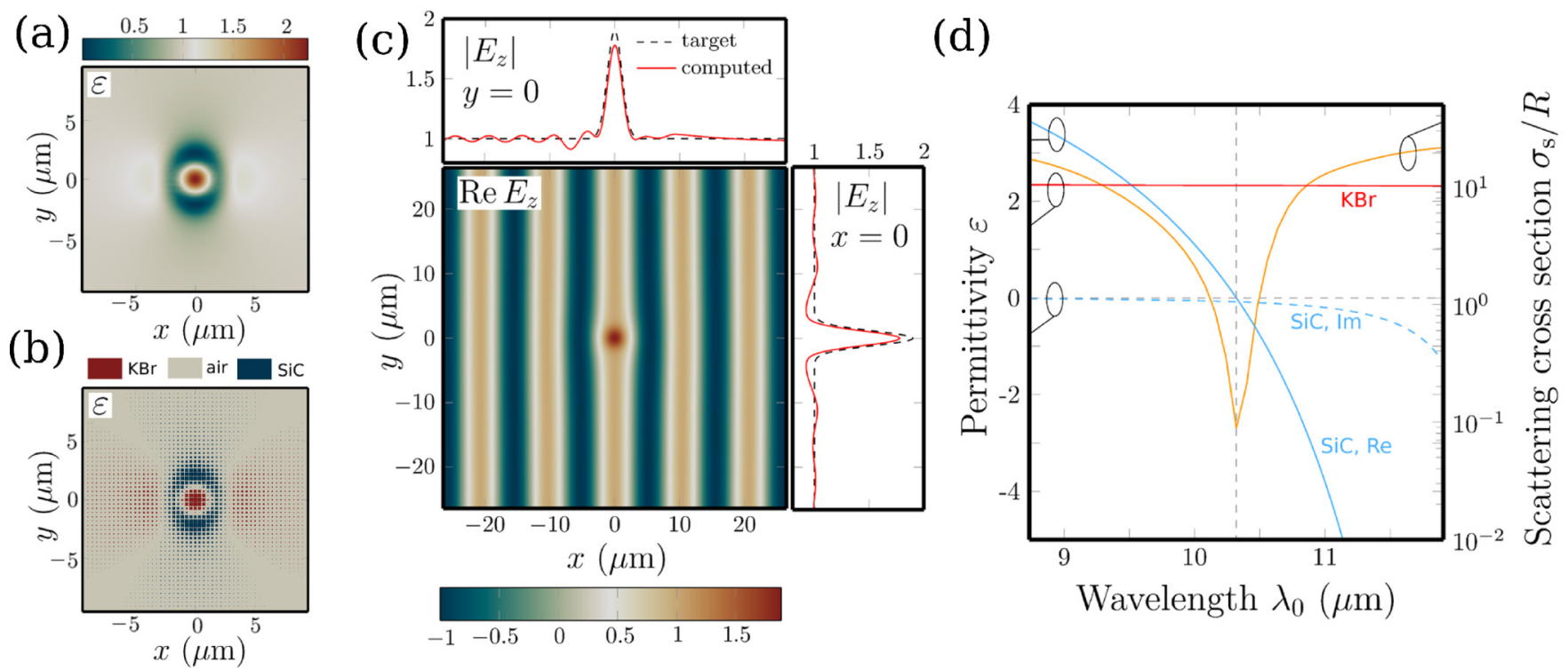

FIG. 4. Invisible metamaterial with subwavelength control of the amplitude. (a) Continuous and (b) metamaterial permittivity profiles. (c) Central color map: real part of the electric field at $\lambda_{0}=10.32 \mu \mathrm{m}$, top and right panels: target (black dashed lines) and calculated (red solid lines) amplitudes for $y=0$ and $x=0$, respectively. (d) Left ordinate axis: permittivity dispersion of SiC (solid and dashed cyan lines for real and imaginary parts) and $\mathrm{KBr}$ (solid red line); the horizontal dashed line indicates a zero value; right ordinate axis: scattering cross section spectra of the metamaterial structure. The vertical dashed line indicates $\lambda_{0}=\lambda_{\mathrm{L}}=10.32 \mu \mathrm{m}$ at which we designed the structure.

values $\varepsilon_{i j}=\varepsilon\left(x_{i}, y_{j}\right)$. Since the period is much smaller than the wavelength, we can safely use an effective permittivity $\varepsilon_{\text {eff }}$ given by the Maxwell-Garnett homogenization formula:

$$
\left(\frac{\varepsilon_{\mathrm{eff}}-\varepsilon_{\mathrm{h}}}{\varepsilon_{\mathrm{eff}}+2 \varepsilon_{\mathrm{h}}}\right)=f\left(\frac{\varepsilon_{\mathrm{i}}-\varepsilon_{\mathrm{h}}}{\varepsilon_{\mathrm{i}}+2 \varepsilon_{\mathrm{h}}}\right),
$$

where $\varepsilon_{\mathrm{h}}$ is the permittivity of the host medium (air in our case), $\varepsilon_{\mathrm{i}}$ is the permittivity of the inclusions (either $\mathrm{SiC}$ or $\mathrm{KBr}), f=a^{2} / d^{2}$ is the filling fraction, and $a$ is the length of the square section of the rods. The structure is then constructed as follows: if $\varepsilon_{i j}<0.99$ we use $\mathrm{SiC}$ rods, if $\varepsilon_{i j}>1.01$ we use $\mathrm{KBr}$ rods, otherwise we just use air [see Fig. 4(b)]. The real part of the electric field is plotted in Fig. 4(c), and clearly illustrates the invisibility effect and the subwavelength control of the amplitude. The top and left panels compare the target (black dashed lines) and calculated (red solid lines) amplitudes for $y=0$ and $x=0$, respectively, revealing a quasiperfect match apart from a small scattering, mostly due to the truncation and discretization of the permittivity profile and a slightly weaker amplitude than expected, due to losses in SiC rods. The scattering cross section spectrum in Fig. 4(d) exhibits a pronounced dip around $\lambda_{0}=10.32 \mu \mathrm{m}$, which illustrates the strong reduction of diffraction resulting in a quasi-invisible complex metamaterial.

\section{THE INVERSE PROBLEM: CONTROLLING AMPLITUDE AND PHASE}

Finally, we study the inverse problem of finding invisible material properties that give a predefined electric field. To this aim, we fix the amplitude $A$ and the additional phase term $\psi$ and rewrite Eq. (2) as

$$
A^{2} \nabla \phi \cdot \nabla u=\nabla \cdot\left(A^{2} \nabla \phi\right),
$$

with $u=\ln \mu$. This equation is then solved numerically and the obtained value of $\mu$ is plugged into Eq. (3) to obtain $\varepsilon$.

For the following example, we set $\lambda_{0}=700 \mathrm{~nm}, R=\lambda_{0}$, $\theta_{0}=\pi / 3$,

$$
\begin{aligned}
A= & 1-0.3 e^{-\left[\left(x-2 \lambda_{0}\right)^{2}+0.5\left(y+2 \lambda_{0}\right)^{2}\right] / R^{2}} \\
& +0.4 e^{-\left[0.6\left(x+2 \lambda_{0}\right)^{2}+\left(y-2 \lambda_{0}\right)^{2}\right] / R^{2}}
\end{aligned}
$$

and

$$
\psi=k_{0}\left[x_{a}^{\prime \prime} e^{-\left[x_{a}^{\prime \prime 2}+0.4 y_{a}^{\prime \prime 2}\right] / R^{2}}-0.7 x_{b}^{\prime \prime} e^{-\left[0.5 x_{b}^{\prime \prime 2}+y_{b}^{\prime \prime 2}\right] / R^{2}}\right]
$$

using the shifted and rotated coordinates:

$$
\begin{aligned}
x_{a}^{\prime \prime} & =n_{x} x_{a}^{\prime}+n_{y} y_{a}^{\prime}, & x_{a}^{\prime} & =x-2 \lambda_{0}, \\
y_{a}^{\prime \prime} & =-n_{y} x_{a}^{\prime}+n_{x} y_{a}^{\prime}, & y_{a}^{\prime} & =y-2 \lambda_{0}, \\
x_{b}^{\prime \prime} & =n_{x} x_{b}^{\prime}+n_{y} y_{b}^{\prime}, & x_{b}^{\prime} & =x+2 \lambda_{0}, \\
y_{b}^{\prime \prime} & =-n_{y} x_{b}^{\prime}+n_{x} y_{b}^{\prime}, & y_{b}^{\prime} & =y+2 \lambda_{0} .
\end{aligned}
$$

This particular choice of amplitude and phase will give the following wave behavior: amplitude damping at $\left(+2 \lambda_{0},-2 \lambda_{0}\right)$, amplitude enhancement at $\left(-2 \lambda_{0},+2 \lambda_{0}\right)$, phase expansion at $\left(-2 \lambda_{0},-2 \lambda_{0}\right)$, and phase compression at $\left(+2 \lambda_{0},+2 \lambda_{0}\right)$ [see Figs. 5(e), 5(g), and 5(c) for the specified amplitude, additional phase, and electric field, respectively]. The obtained values of material properties are plotted in Fig. 5(a) for the permittivity and Fig. 5(b) for the permeability. These nontrivial profiles allow us to control the wave propagation quite arbitrarily in the near field while being transparent to a specific incident plane wave. Note that as stated before, the same profiles are still invisible for a wave coming from the opposite direction, and maintain the amplitude control but the phase now has opposite sign.

To double-check the validity of our results, we solved the wave equation (1) employing the permittivity and permeability 


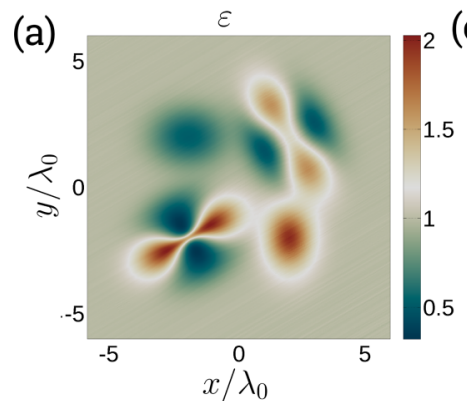

(b)

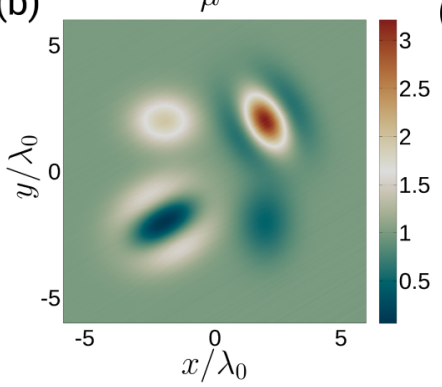

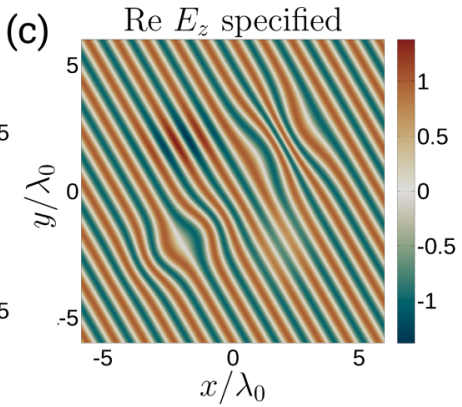

(d)

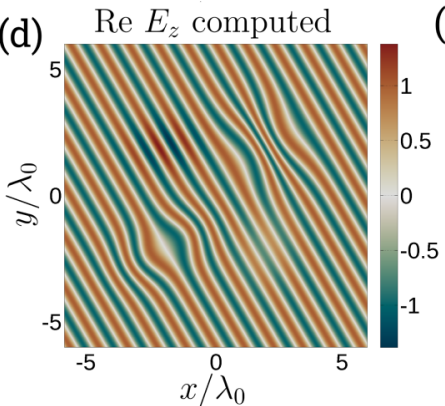

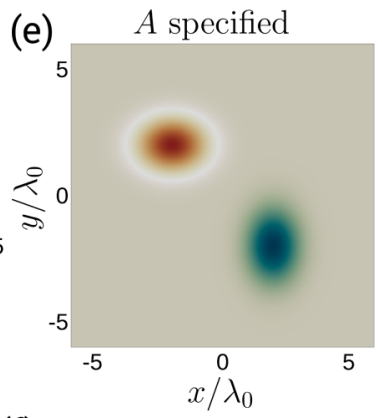

(f)

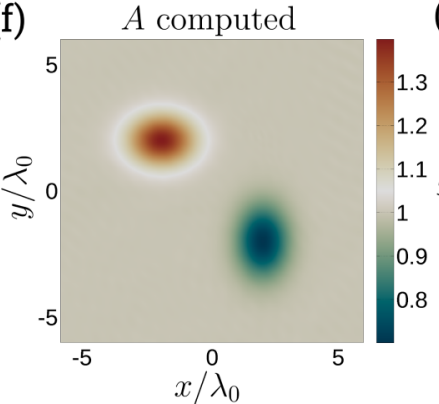

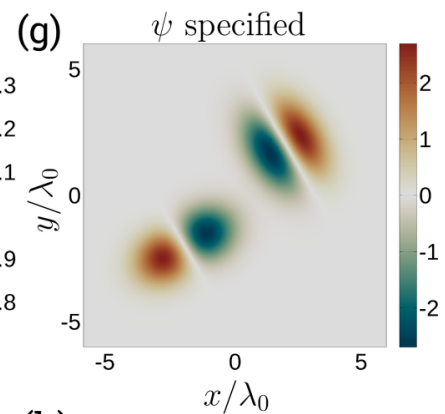

(h)

$\psi$ computed

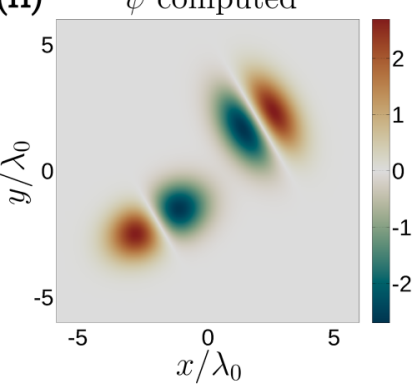

FIG. 5. Inverse design of amplitude and phase profiles (see text for definitions) represented in (e) and (g), giving a desired electric field (c). Required permittivity (a) and permeability (b) are the used to solve the wave equation (direct problem) for a sanity check of the field (d), amplitude (f), and phase (h).

obtained by our approach. The results are plotted in Figs. 5(f), 5(h), and 5(d) for the amplitude, additional phase, and electric field, respectively, and match the required wave behavior perfectly. The generality of this inverse problem makes it quite versatile and reveals a family of amplitude and phase controlling invisible electromagnetic media.

\section{CONCLUSION}

In conclusion, we have presented a flexible and systematic methodology to derive isotropic and lossless material properties needed to manipulate the amplitude and phase of the electromagnetic field in an arbitrary way, for planar propagation. In addition, our work provides a contribution in the understanding of what governs scattering in this type of media. Since it is based on the scalar wave equation, it could be easily extended to other fields such as acoustics or fluid dynamics. In particular, we have applied this method to derive a large class of invisible permittivity and permeability profiles. We illustrated these concepts through numerical examples for TE polarized plane waves using both $\varepsilon$ and $\mu$ and obtained omni-directional invisibility and control of the amplitude. Then we studied the case of nonmagnetic materials and showed that one can obtain invisibility and fashion the spatial variation of the magnitude of the electric field for two antiparallel directions of incidence. A metamaterial structure working in the infrared has been proposed, exhibiting subwavelength control of waves and invisibility at the same time. Finally, we tackled the inverse problem of finding nonscattering material properties that give a specified electric field. These results pave the way for a new route towards achieving invisibility with isotropic materials, and may offer an alternative paradigm for the design of nanophotonic devices with enhanced performances.

\section{ACKNOWLEDGMENTS}

This work was funded by the Engineering and Physical Sciences Research Council (EPSRC), UK, under a Programme Grant (Grant No. EP/I034548/1) "The Quest for Ultimate Electromagnetics using Spatial Transformations (QUEST).”
[1] J. B. Pendry, D. Schurig, and D. R. Smith, Science 312, 1780 (2006).

[2] U. Leonhardt and T. G. Philbin, New J. Phys. 8, 247 (2006).

[3] U. Leonhardt, Science 312, 1777 (2006).

[4] D. Schurig, J. J. Mock, B. J. Justice, S. A. Cummer, J. B. Pendry, A. F. Starr, and D. R. Smith, Science 314, 977 (2006).

[5] J. Valentine, J. Li, T. Zentgraf, G. Bartal, and X. Zhang, Nat. Mater. 8, 568 (2009).

[6] T. Ergin, N. Stenger, P. Brenner, J. B. Pendry, and M. Wegener, Science 328, 337 (2010).

[7] H. Chen, C. Chan, and P. Sheng, Nat. Mater. 9, 387 (2010).
[8] O. Quevedo-Teruel, W. Tang, and Y. Hao, Opt. Lett. 37, 4850 (2012).

[9] O. Quevedo-Teruel, W. Tang, R. C. Mitchell-Thomas, A. Dyke, H. Dyke, L. Zhang, S. Haq, and Y. Hao, Sci. Rep. 3, 1903 (2013).

[10] A. Alù, Phys. Rev. B 80, 245115 (2009).

[11] J. Andkjær and O. Sigmund, Appl. Phys. Lett. 98, 021112 (2011).

[12] B. Vial and Y. Hao, Opt. Express 23, 23551 (2015).

[13] Z. Lin, H. Ramezani, T. Eichelkraut, T. Kottos, H. Cao, and D. N. Christodoulides, Phys. Rev. Lett. 106, 213901 (2011).

[14] A. Mostafazadeh, Phys. Rev. A 87, 012103 (2013). 
[15] M. V. Berry and C. J. Howls, J. Phys. A: Math. Gen. 23, L243 (1990).

[16] S. A. R. Horsley, C. G. King, and T. G. Philbin, J. Opt. 18, 044016 (2016).

[17] T. G. Philbin, J. Opt. 18, 01 LT01 (2016).

[18] S. Horsley, M. Artoni, and G. La Rocca, Nat. Photonics 9, 436 (2015).

[19] S. Zeng, D. Baillargeat, H.-P. Ho, and K.-T. Yong, Chem. Soc. Rev. 43, 3426 (2014).

[20] R. Singh, W. Cao, I. Al-Naib, L. Cong, W. Withayachumnankul, and W. Zhang, Appl. Phys. Lett. 105, 171101 (2014).

[21] E. Ozbay, Science 311, 189 (2006).

[22] M. Li, W. Pernice, C. Xiong, T. Baehr-Jones, M. Hochberg, and H. Tang, Nature (London) 456, 480 (2008).

[23] R. Zhang, Y. Zhang, Z. Dong, S. Jiang, C. Zhang, L. Chen, L. Zhang, Y. Liao, J. Aizpurua, Y. Luo et al., Nature (London) 498, 82 (2013).

[24] P. L. Stiles, J. A. Dieringer, N. C. Shah, and R. P. Van Duyne, Annu. Rev. Anal. Chem. 1, 601 (2008).
[25] P. Genevet, J.-P. Tetienne, E. Gatzogiannis, R. Blanchard, M. A. Kats, M. O. Scully, and F. Capasso, Nano Lett. 10, 4880 (2010).

[26] H. Harutyunyan, G. Volpe, R. Quidant, and L. Novotny, Phys. Rev. Lett. 108, 217403 (2012).

[27] M. Kauranen and A. V. Zayats, Nat. Photonics 6, 737 (2012).

[28] C. Höppener, Z. J. Lapin, P. Bharadwaj, and L. Novotny, Phys. Rev. Lett. 109, 017402 (2012).

[29] C. Belacel, B. Habert, F. Bigourdan, F. Marquier, J.-P. Hugonin, S. Michaelis de Vasconcellos, X. Lafosse, L. Coolen, C. Schwob, C. Javaux et al., Nano Lett. 13, 1516 (2013).

[30] P. R. Holland, The Quantum Theory of Motion: An Account of the de Broglie-Bohm Causal Interpretation of Quantum Mechanics (Cambridge University Press, Cambridge, UK, 1995).

[31] T. Philbin, J. Mod. Opt. 61, 552 (2014).

[32] J. Lekner, Am. J. Phys. 75, 1151 (2007).

[33] E. D. Palik, Handbook of Optical Constants of Solids (Academic, New York, 1991).

[34] H. Li, J. Phys. Chem. Ref. Data 5, 329 (1976). 